\title{
Type 2 diabetes mellitus, physical activity, yoga and telomere length: A literature review
}

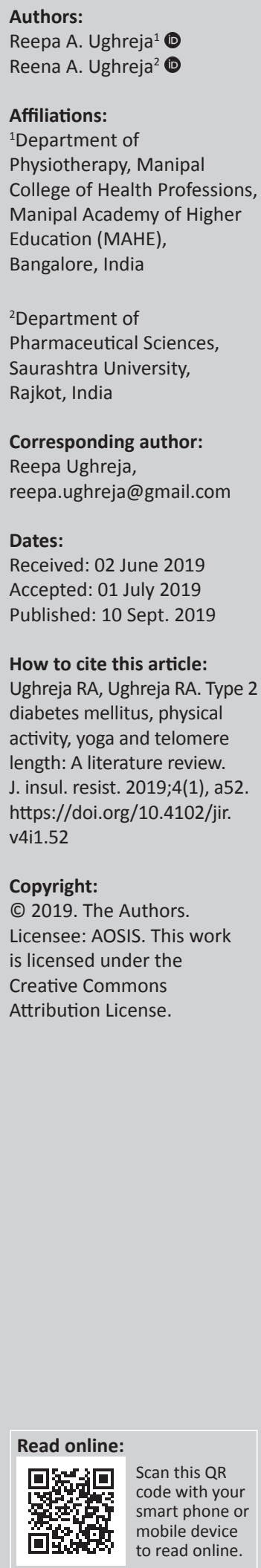

Background: Type 2 diabetes mellitus (DM), a chronic metabolic disease prevalent in adults, is also prevalent amongst children, adolescents and young adults. On understanding the molecular basis of diabetes, a significant association is found between telomere length (TL) and type $2 \mathrm{DM}$.

Aim: The aim of the study was to review the available evidence on effect of physical activity and yoga on DM including their effect on TL.

Setting: The study was conducted in Bangalore.

Method: A number of databases such as Google Scholar, PubMed and Cochrane Review were searched for relevant articles using keywords such as 'diabetes', 'type $2 \mathrm{DM}^{\prime}$, 'physical activity', 'yoga', 'TL' and 'telomerase activity'. All types of articles were included for the study, such as randomised controlled trial, systematic reviews, literature review and pilot study. Non-English articles were excluded from the study.

Results: Studies have demonstrated the effectiveness of yoga and physical exercise in type 2 $\mathrm{DM}$ in various ways, such as reducing fasting blood glucose and glycosylated haemoglobin; improving lipid profile, blood pressure and waist-to-hip ratio; reducing inflammatory, oxidative and psychological stress markers; and improving the quality of life of patients. However, limited information is available on the effect of these interventions on TL in type 2 DM and mechanisms involved.

Conclusion: Recent studies have shown positive effects of yoga and physical activity on TL. However, there is a dearth of good-quality studies evaluating the effects of yoga on TL in type 2 DM. Future studies need to be conducted with standard treatment protocols, long-term follow-up, appropriate control groups and large sample size.

Keywords: Type 2 Diabetes Mellitus; Yoga; Physical Activity; Telomere Length; Quality of Life.

\section{Introduction}

Diabetes mellitus (DM) is a chronic disease characterised by higher blood glucose levels because of insufficient production of insulin hormone or ineffective use of insulin. ${ }^{1}$ As per the report of International Diabetes Federation diabetes atlas (8th edition, 2017), 425 million adults aged between 20 and 79 years in the world have diabetes, a number that is anticipated to rise to 629 million people by 2045. It was found that the maximum numbers of persons with diabetes were adults between 40 and 59 years of age and that $79 \%$ adults having diabetes live in low- and middle-income countries. This shows higher prevalence of diabetes in countries such as India (74 million people) and China (121 million people). ${ }^{2}$

Diabetes mellitus is classified into three main categories namely: type 1, type 2 and gestational diabetes. Out of the three, type $2 \mathrm{DM}$ is the commonest type that accounts for approximately $90 \%$ of all the cases of diabetes in adults. ${ }^{2}$ It is more frequently found in elder adults but it has been also commonly seen nowadays in children, adolescents and younger adults because of poor lifestyle factors such as increasing levels of sedentary activity, improper diet and obesity. ${ }^{2}$ Type 2 DM is characterised by hyperglycaemia, insulin resistance and other metabolic irregularities including high blood pressure (BP), abnormal lipid profiles, chronic inflammation, excessive coagulation, excessive oxidative stress, and so on..$^{3,4,5,6,7}$ Diabetes mellitus is one of the leading causes of morbidity and mortality and increases the risk for various complications such as atherosclerosis, retinitis, neuropathy, renal disease, depression, dementia, cardiovascular diseases, and so on. ${ }^{3,4,8}$

On understanding the molecular basis of diabetes, a significant association is found between telomere length (TL) and type 2 DM. ${ }^{9}{ }^{10}$ In a meta-analysis, it was found that TL is reduced in type 
$2 \mathrm{DM}$ patients as compared to healthy people. ${ }^{9}$ It also stated that TL is also affected by geographical area, age, gender, type of DM and body mass index (BMI) (especially in obesity). ${ }^{9}$ Telomere length is also found to be influenced by oxidative stress which is one of the major contributors for DM. ${ }^{11}$ Thus, understanding mechanisms by which DM affects TL can help in preventing and treating DM.

An important part of management in DM not only includes the treatment of high glucose but also the management of hyperlipidaemia, hypertension, body weight by maintaining a healthy lifestyle that includes a healthy nutritious diet, increased physical activity and cessation of smoking with appropriate use of medications. ${ }^{1,12,13,14}$ The treatment also aims at reducing depression, agony and improving the quality of life (QOL) of the patient which, in turn, affects the glycaemic control, the rate of complications, treatment compliance, and so on. ${ }^{8}$ Thus, keeping in mind the above, various alternative treatment options and lifestyle interventions are also used for the management of DM..$^{15,16,17}$ These include yoga, meditation, dietary supplements, acupuncture, massage, hydrotherapy, aerobic and resistance exercises and so on. Studies have demonstrated the effectiveness of yoga and meditation in type $2 \mathrm{DM}$ in various ways such as reducing fasting blood glucose (FBG) and glycosylated haemoglobin, improving lipid profile, improving BP and waist-to-hip ratio, reducing inflammatory, oxidative and psychological stress markers, improving depression and cognitive task performance, thus improving the QOL of patients. ${ }^{12,18,19,20,21,22,23,24,25}$ Physical activities like aerobic and resistance exercises have also shown improvements in type $2 \mathrm{DM}$ patients by improving glycaemic control and reducing the risk factors linked with cardiovascular pathologies. . $^{1526,27}$

The purpose of this study was to review analytically the data available on the effectiveness of yoga and other physical activities in type $2 \mathrm{DM}$ patients. It also aimed to understand and outline the underlying mechanisms responsible for improvement in these patients. The review also attempted to evaluate the effect of yoga on TL from the available evidence and ascertain its implication in type $2 \mathrm{DM}$.

\section{Methods}

The articles included for the study were searched from various databases such as Google Scholar, PubMed and Cochrane. The keywords used for the search were diabetes, type $2 \mathrm{DM}$, physical activity, yoga, TL, telomerase activity and so on. All types of articles were included for the study such as randomised controlled trial, systematic reviews, literature review and pilot study. The articles in language other than English were excluded. The limitation of the method used was that quality analysis and risk of bias assessment was not carried out.

\section{Review findings}

Following were the findings of the review:

\section{Physical activity and type $\mathbf{2}$ diabetes mellitus}

Physical activity is one of the important components in the management of diabetes. Physical inactivity is one of the important modifiable risk factors for type $2 \mathrm{DM}^{2}$ According to the updated guidelines by American Diabetes Association (ADA) and American College of Sports Medicine (ACSM), 150 min of moderate $(50 \%-70 \%$ of a person's maximum heart rate) to vigorous ( $>70 \%$ of maximum heart rate) physical activity per week is recommended for patients with type $2 \mathrm{DM}^{28}$ Various studies have shown the effectiveness of physical activities on glycaemic control, lowering of glycated haemoglobin (HbA1c) levels, fat mass, triglycerides, lipid levels and reduction of risk factors for cardiovascular disease. ${ }^{8,15,26,29,30,31,32,33,34,35}$ Amongst these physical activities, yoga, aerobic exercises and resistance exercises were found to be more effective than other physical activities. ${ }^{28,30,35}$

In a systematic review and meta-analysis on diabetic patients over 18 years of age, regular physical activity in the form of aerobic and resistance exercises (for 8 weeks or more) were effective in reducing blood sugar level in the short term, and in the long term, the levels of $\mathrm{HbA1c}$ were significantly reduced $\left(0.60 \%\right.$ lower than the control group). ${ }^{30}$ It was found that improvements were more in those patients who performed yoga followed by those who were given tai chi walking. Yoga and tai chi (both being an aerobic physical activity) made use of breathing manoeuvres and body movements that burnt calories and lowered $\mathrm{HbA} 1 \mathrm{c}$ levels in the long term. This result was similar to the results found in various other studies that included aerobic physical activities. ${ }^{36,37,38}$ However, in the present study, men showed poor glycaemic control indicating the need to pay more attention for men.

In another study on pre-diabetic individuals (intermediate hyperglycaemia according to World Health Organization [WHO]), the effect of structured exercises (according to ADA guidelines) on sedentary obese individuals (less than twice per week of aerobic exercise) was evaluated. ${ }^{26}$ It showed that resistance and aerobic training had good improvements on impaired fasting glucose (IFG) and impaired glucose tolerance (IGT) in pre-diabetics and those with diabetes had restoration to normal glucose levels. However, interval training was not evaluated. The study suggests the prediabetics to be included in the diabetes prevention programme as compared to those with overall risk factors for type $2 \mathrm{DM}$ because they are benefitted more than those with DM. A consensus statement from ADA also showed that physical activity in the form of aerobic and resistance exercise and diet-induced weight loss (by 5\% - 7\%) reduced the risk of progression from IGT to type 2 DM by $58 \%{ }^{35}$

A pilot study showed that 6 weeks of functional highintensity crossfit training (three sessions of resistance training, gymnastics and aerobic exercises for 10-20 min) improved function of beta cells by reducing fasting proinsulin or insulin ratio (responsible for the processing inefficiency of insulin), decreased abdominal fat and improved liver function. Abdominal fat is connected to 
insulin resistance which, in turn, relates to beta cell dysfunction. This study can be an effective approach to improve the adherence to the treatment plan in type $2 \mathrm{DM}$ patients because of structured programme for a short time period. Nevertheless, given the differential response to exercise, further studies need to perform to devise the most effective exercise regimen. ${ }^{39}$

In a systematic review by Hemmingsen et al., the effect of diet or physical activity alone or effects of both were evaluated. It was concluded that there was no effect of diet or physical activity alone on the risk of type $2 \mathrm{DM}$ on people at high risk of developing type $2 \mathrm{DM}$. Nonetheless, diet plus physical activity together reduced or delayed the incidence of type $2 \mathrm{DM}$ in people with IGT. However, the effects on complications of DM and prediabetes were not investigated. ${ }^{34}$

Another study examined the effect of lifestyle interventions on the risk factors for cardiovascular disease in type $2 \mathrm{DM}$. These interventions included changes in diet, exercise and education (at least one of smoking cessation, behaviour modification, counselling and so on) for more than 6 months duration. Results showed significant improvements in BMI, $\mathrm{HbA1c}$, systolic and diastolic BP (all $p=0.014$ ) except any improvements in high- and low-density lipoprotein levels. But the limitations of the study were that there was a high risk of bias in the blinding of subjects and evaluators and effect of specific exercise was not evaluated. ${ }^{15}$

Apart from the structured physical activity, studies have proven efficacy of daily physical activity too. Daily physical activity includes different activities carried out during both leisure time as well as during work. For example, walking, gardening and household activities that can be easily performed by type $2 \mathrm{DM}$ patients. A review by Hamasaki ${ }^{29}$ found that walking that was the commonest form of daily physical activity exhibited benefits on lowering the risk of type $2 \mathrm{DM}$, cardiovascular disease and mortality. Walking for at least 30 min a day demonstrated type 2 DM risk reduction by $50 \%$. Nevertheless, there was scarce literature about the efficacy of other daily physical activities like gardening and housework. Non-exercise activity thermogenesis was also found to be associated with metabolic risks and mortality, but further studies are required to demonstrate its effects on the health of the patients.

A study showed that diabetic patients encounter various facilitators (awareness of benefits of exercise and complications of DM and family support) and barriers (time constraints, lack of perception of obesity as a health issue, inability to associate exercise with blood sugar control, sociocultural issues, improperly trained diabetic educators) to an exercise programme. These should be taken into account to improve the knowledge and adherence to the exercise programme. ${ }^{40}$ Another study showed lack of knowledge, fear of production or exacerbation of symptoms and lack of exercise venue as the barriers to exercise in addition to the above factors. ${ }^{41}$ In a study, it was found that exercises performed through the use of activity monitor were more effective over pedometer in reducing $\mathrm{HbA} 1 \mathrm{c}$ levels at 2 months. The activity monitor motivated the patient to continue exercise at moderate level along with giving feedback to the patient about the exercise intensity. ${ }^{42}$

In summary, it can be concluded that physical exercise has a significant role in improving glycaemic control and reducing the risk of cardiovascular disease in patients with type $2 \mathrm{DM}$. However, there was a problem in patient adherence to the treatment programme. Lower physical performance, lowenergy expenditure, cardiorespiratory fitness and muscle strength inversely associate with the degree of diabetic complications. ${ }^{29}$ This further hinders the involvement in physical activity. The review suggests to conduct studies that include pre-diabetics for the diabetes prevention programme.

\section{Recommendations of physical activity}

Following are the updated guidelines on exercise in diabetes: 28,31

Aerobic exercise: $150 \mathrm{~min}$ or more of moderate to vigorous activity per week for at least three or more days a week.

High-intensity interval training: Short bursts of highintensity exercise with retrieval period either in the form of rest or an activity of low-intensity results in better insulin sensitivity and better overall blood glucose levels.

Resistance exercises: Variety of strength training on nonconsecutive days for two to three sessions per week. Heavy training has a better impact on glycaemic control, strength, balance and ability to live life self-sufficiently than lighter exercises or home-based activities. Resistance training followed by aerobic exercise helps to maintain the glycaemic balance.

Flexibility and balance exercises: Performed two to three times per week. Stretching exercises help to improve the range of motion and gait and balance training prevents falls in elderly. Yoga improves glycaemic control, lipid levels and body composition in adults. Tai chi training helps to improve glycaemic control, stabilises symptoms of neuropathy and improves the QOL of the patients.

Daily unstructured physical activity: Such as household tasks, gardening, dog walking, and so on, helps in losing and maintaining weight, reducing post-prandial blood sugar.

Aerobic exercises are better over resistance training in terms of improvement in glycaemic control. Both have similar effect over the cardiovascular risk markers. The combined effect of both the exercises leads to better management of blood glucose.

\section{Mechanism behind the efficacy of physical activity in type 2 diabetes mellitus}

Aerobic as well as resistance exercises have shown to increase muscle GLUT4. This increase in GLUT4 probably leads to the increased capacity for insulin-stimulated glucose transport in 
trained subjects. ${ }^{43,44}$ This theory has imperative implications for people with insulin resistance. Exercise training has also shown to stimulate insulin-stimulated PI 3-kinase in muscle. This increase is thought to be because of IRS-1-associated PI 3-kinase activity. As PI 3-kinase is an important step in the recruitment of GLUT4 by insulin to the muscle cell surface, it can be postulated that at this step, regular physical activity may affect insulin signalling. Regular physical activity leads to an increase in basal and insulin-stimulated Mitogenactivated protein (MAP) kinase pathway activity. Type 2 diabetes patients with low insulin secretion have shown a rise in the insulin secretion because of physical training. This can be because of sensitisation of the autonomic nervous system along with it. Peripheral insulin concentrations are not altered, suggesting that the extra insulin produced is captured by the liver. ${ }^{45}$ The mechanisms through which aerobic and resistance exercise increase glucose disposal are similar. Although resistance exercise has greater tendency than aerobic exercise to increase muscle mass and hence glucose storage space, this is only one of many factors explaining its effects on glucose disposal. ${ }^{43}$

\section{Yoga and type 2 diabetes mellitus}

The term 'Yoga' is derived from the Sanskrit word yug which means union of individual's consciousness with the divine consciousness. ${ }^{16}$ Yoga originated more than 4000 years ago and has become very popular in both developed and developing countries all over the world. ${ }^{8,19}$ It not only focuses on physical fitness but also on integration of body, mind and soul, creation of harmony, awareness, balance, cultivation of selflessness and spiritual enlightenment. ${ }^{46}$ Out of the various branches of yoga, some of the commonly used forms include Raja yoga and Hatha yoga (yoga of activity). ${ }^{8}$ These Raja and Hatha yogas have shown to improve health and reduce illness by asanas (both active and passive relaxation postures), pranayama (breathing control), dharana (concentration) and dhyana (meditation). ${ }^{8,47}$ Yoga has become one of the increasingly used adjunctive therapies in the treatment of type $2 \mathrm{DM}^{48}$ Various studies have shown the efficacy of yoga on glycaemic control, improvement in blood glucose, cholesterol, oxidative stress markers, BP, anthropometric measurements, heart rate, sympathetic activity, coagulation profiles, pulmonary function, reduction in the risk factors for cardiovascular disease and thus its contribution towards health in patients with diabetes. $8,12,18,19,20,21,22,23,24,25,49$

A systematic review of controlled trials performed on the effect of yoga on type 2 DM revealed that yoga produced significant effect on various parameters in DM treatment and prevention. These factors include glycaemic control (FBG, postprandial blood glucose [PPBG], fasting insulin and $\mathrm{HbA1c})$, insulin resistance, reduction in low-density lipoprotein (LDL) and increase in high-density lipoprotein (HDL), reduction in waist-to-hip ratio and reduction in BP. Yoga is also thought to reduce oxidative stress, reduce sympathetic activation, enhance nervous system function, pulmonary performance, mood, sleep, QOL and decrease usage of medicines. But there are very few studies performed on the above parameters and very few studies on effect of yoga on risks (including pro-inflammatory markers) and complications in type 2 DM. Moreover, the dosage of yoga and the programme specifications varied much amongst the studies. Thus, this study suggests controlled trials of high quality including standard treatment programmes and to be carried out in various countries other than India (as most were Indian trials).

Another systematic review and meta-analysis concluded similar results on the beneficial effects of yoga on FBG, PPBG and $\mathrm{HbA} 1 \mathrm{c}$ levels. ${ }^{12}$ There were, nonetheless, limitations such as publication bias, high risk of bias, usage of medicine, small sample size and short follow-up. Hence, the study showed that yoga is an efficient complementary treatment for blood glucose parameters in type $2 \mathrm{DM}$ patients for the short term. Future studies should focus on good quality with large sample size with long-term follow-up and making use of outcomes measures other than the blood glucose parameters.

In another meta-analysis, similar results were obtained. Yoga significantly reduced levels of FBG, PPBG, HbA1c, total cholesterol (TC), LDL and increased the levels of HDL. Henceforth, the study suggested that yoga can be considered as a possible alternative management for type 2 DM. However, the studies varied much in terms of participant characteristics, parameters and the forms of yoga. Hence, further studies are required to determine the appropriate exercise programme and long-term effects of yoga with the help of objective measurements such as QOL, BP, inflammatory mediators and immune cell function. ${ }^{18}$

A recent review also showed the beneficial effects of yoga on blood glucose levels (FBG, PPBG, usage of anti-diabetic drugs and management of comorbid diseases in type $2 \mathrm{DM}$ patients). The study stated that yoga helps in prevention and control of DM, control of body weight, BP and lipid profile, prevention of cardiovascular events, complications of DM, pancreatic regeneration and exercise tolerance. It also helped to improve the QOL, mood and stress levels, coagulation profile and cognitive function of patients. However, the study lacked proper control groups, appropriate sample size, appropriate duration, proper methodology, and so on. Hence, further studies need to improve methodology, reduce bias and study long-term effects of yoga. ${ }^{19}$ The possible mechanisms responsible for the beneficial effects discussed in the study were psychological, neurological, endocrine and immune mechanisms. Furthermore, parasympathetic activation and associated anti-stress mechanism improved the metabolic and psychological profiles, insulin sensitivity, IGT and lipid profiles. These results were similar to a previous review performed in 2015 by Singh et al. ${ }^{21}$

In another systematic review and meta-analysis, the studies included had a control group, reported an objective measure of glycaemic control and had follow-up of at least 8 weeks. The study showed that yoga had significant improvement in glycaemic outcomes (HbA1c, FBG and PPBG), lipid profiles, 
BP, BMI, waist-to-hip ratio and cortisol levels. ${ }^{22}$ These findings were similar to those of other meta-analyses. ${ }^{12,18}$ The subjects from non-clinical setting showed a greater reduction in FBG, TC and LDL than those from clinical settings (possibility of the subjects from clinics having better baseline glucose levels). Home programmes showed lesser improvements in FBG, LDL and HDL. The reason could be poor adherence, short duration of the study or both. Meditation techniques were effective in reducing weight and triglyceride levels, whereas relaxation techniques helped in stress reduction. Moreover, most of the studies were performed in India. Hence, the patients might be familiar with the yoga forms. In spite of all the above, the study had a few limitations of poor quality of methodology and absence of long-term follow-up. The study details were not sufficient; there were various yoga components. The yoga style was not described and individual session duration was not mentioned. Thus, the study suggests to conduct standard randomised trials with long-term follow-up.

A randomised controlled trial on 80 subjects (35-55 years old) evaluating the effect of yogasanas and pranayama in type 2 DM patients (with disease duration ranging from 1 to 10 years) found that there were significant reductions in the levels of FBG, PPBG and HbA1c $(p<0.001)$ as compared to control group. ${ }^{23}$ The intervention that was continued for 3 months included 13 yogasanas and pranayam, each for 45-60 min, 5 days a week for 12 weeks. Oral anti-diabetic drugs were given to both the groups. The possible mechanisms thought were as follows: (1) Abdominal stretching during yoga causes direct regeneration of beta cells of pancreas which increases utilisation and metabolism of glucose in peripheral tissues, liver and adipose. (2) Yogic exercises lead to deeper relaxation and possibility of neuroplasticity that may bring about changes in the hypothalamic-pituitary-pancreatic axis. (3) Muscle relaxation and improved blood circulation increase the insulin receptor expression activity and thus increase glucose uptake and thus reduce the blood glucose. The findings of this study were similar to various studies performed previously. ${ }^{50,51}$

In another randomised trial, ${ }^{52}$ the effect of 4 weeks yoga intervention on FBG and PPBG was evaluated in 30 patients with type $2 \mathrm{DM}$. The result showed that there were no significant differences in FBG in the yoga group $(p=0.31)$. However, a significant difference was seen on PPBG in yoga group ( $p=0.0015) .^{52}$ The control group showed no significant differences in either of the two. These findings were not consistent with the results seen in a similar study performed by Chimkode et al., who found that 3-6 months of yoga significantly reduced blood glucose levels. ${ }^{53}$ The reason for this inconsistency between the two studies was thought to be because of the different set of yogasanas in the exercise programme. The former study included $20 \mathrm{~min}$ of yognidra (a stress relaxation technique). Henceforth, even a short duration of yoga (4 weeks) was found to be effective in reducing the glucagon secretion. ${ }^{52}$
In conclusion, yoga is an effective alternative or complementary treatment in type $2 \mathrm{DM}$. However, future studies should focus on conducting high-quality studies with proper control groups, appropriate methodology of yoga interventions, appropriate (large) sample size, patient adherence to the treatment, long-term follow-up and outcome measures other than blood glucose parameters.

\section{Mechanism behind the efficacy of yoga in type 2 diabetes mellitus}

The following are the possible mechanisms responsible for the beneficial effect of yoga in type $2 \mathrm{DM}$.

Firstly, yoga may help to reduce the negative effect of stress and contribute to various positive effects on metabolic, neuroendocrine function and the associated inflammatory reactions. Thus, it helps to lower the risk for cardiovascular diseases by improving the well-being and decreasing the activation of hypothalamic-pituitary-adrenal (HPA) axis and sympathoadrenal system., ${ }^{8,19}$ Studies have shown that a short-term yoga intervention can decrease stress, catecholamine and cortisol levels, improve mood and response of cardiovascular system to stress, BP and other indicators of activation of sympathetic system in both healthy persons and patients including those with type $2 \mathrm{DM}$. It has been found that dysfunction of HPA axis, sympathetic stimulation and stress is associated with the development and exacerbation of various risk factors for type 2 DM. ${ }^{8}$

Secondly, yogic training may cause a shift from sympathetic to parasympathetic stimulation by directly increasing the parasympathetic output probably via vagal stimulation. This results in positive effects on neuroendocrine, haemodynamic and inflammatory markers, sleep and other metabolic parameters. $^{8}$

Thirdly, yoga may also aid in causing desirable changes in autonomic balance, memory, mood, neurological function and other related metabolic and inflammatory responses by activating specific brain structures and neurochemical systems related to attention and positive affect. ${ }^{8}$

In addition, yoga also helps those with type $2 \mathrm{DM}$ indirectly by improving attitudes related to health and lifestyle, providing a source of social support. Yoga is also found to improve flexibility, muscle strength, blood supply and oxygen uptake. ${ }^{54}$ Yoga has various benefits such as improvement in physical fitness, relaxation and selfawareness. Yoga also helps to improve a person's discipline concerning food and exercise thus helping to increase the patient adherence to exercise as a treatment. ${ }^{19}$

The stretching of abdomen during yogic exercise is thought to regenerate $\beta$ pancreatic cells, improving their sensitivity to glucose and improving secretion of insulin. ${ }^{19}$ Yoga also helps to improve muscular blood supply and relaxation thereby improving uptake of glucose. Furthermore, yoga improves immune function by reducing pro-inflammatory responses and cytokines. ${ }^{55}$ 
Yoga also leads to changes in level of hormones and neurotransmitters such as $\beta$ endorphin, serotonin, dopamine, arginine vasopressin, melatonin, and so on. These changes result in joy, euphoria, improvement in arousal, calming effect and blissful feelings, respectively. ${ }^{56}$

Yoga is thought to have the potential to bring stem cell trafficking from bone marrow to the peripheral blood, which, in turn, may contribute to tissue regeneration. Nevertheless, this area needs to be explored further. ${ }^{57}$

Yoga reduces oxidative stress by lowering the levels of malondialdehyde (MDA), interleukin 6 and leptin and improving the levels of adiponectin. ${ }^{55,58}$ Yoga therapy also increases the number of insulin receptors and the proportion of receptor binding in type $2 \mathrm{DM} .{ }^{19}$ Yoga reduces the free fatty acid levels, thus indirectly improving the sensitivity to insulin or reducing the insulin resistance. ${ }^{19}$ Yoga therapy also balances the blood coagulation profile, thus improving nerve conduction and cognitive function in type $2 \mathrm{DM}$ patients.

In an exploratory study, it was concluded that yoga is more advantageous than individual treatments such as physical exercise, caloric restriction and antioxidants. ${ }^{49,59}$ This was thought to be because of the fact that yoga results in energy conservation with economy of energy expenditure for benefits related to mental and physical health and brings about behavioural changes and improvement in cognition and reduction in oxidative stress. ${ }^{60}$ On the other hand, physical exercise leads to energy expenditure more for physical exertion and metabolic needs and results in oxidative stress. ${ }^{61}$ However, because of the absence of homogeneity in the physical exercise regimen, studies have shown the need for future good-quality randomised trials. ${ }^{49}$

\section{Telomere length and type $\mathbf{2}$ diabetes mellitus}

The 'telomere' concept was given by McClintock and Muller. ${ }^{62,63}$ They found that the stability and integrity of the chromosome were retained within the ends of the chromosome, which Muller named the 'telomere'. Thus, the telomere is located at the end of chromosomal deoxyribonucleic acid (DNA) and is composed of thousands of tandem repeats of the DNA sequence (TTAGGG) nucleotide sequence and number of associated proteins, which confer protection against chromosome degradation. ${ }^{64}$ The telomere is essential for DNA replication. It shortens during each DNA replication cycle until it reaches a certain length. At this time, cell apoptosis takes place. Thus, TL is often used as a biological marker for cell ageing. ${ }^{65}$ Accumulating evidence indicates that diabetes may affect changes in TL. Studies have shown an association between type $2 \mathrm{DM}$ and shortened TL. ${ }^{9,10}$ It is probably because of oxidative stress in type $2 \mathrm{DM}$. They suggested that with the development of diabetes, islet $\beta$ cells undergo senescence or apoptosis, because the TL gradually becomes shorter. This leads to decreased functioning of islet cells, which, in turn, can lead to a cascade of damaging events characteristic of diabetes complications. Studies have shown that in type 2
DM, both the leucocyte TL as well as $\beta$ cell TL are reduced. ${ }^{10}$ A meta-analysis found that the TL was not only affected by diabetes type, but also by geographical area, age, BMI and gender. $^{9}$ Type 1 DM obese female patients above 60 years of age had reduced TL as compared to male patients with type 2 DM below 60 years of age. Poor socioeconomic status was also associated with reduced TL because of increased oxidative stress, bad nutrition, unhealthy attitude and physical, emotional and psychological pressure. Contrasting evidences were found in TL with respect to age. ${ }^{9}$ Telomere dynamics are also found to be affected by unhealthy diet, occupational and environmental exposure to toxic agents. ${ }^{10}$

\section{Measurement of telomere length}

Telomere length can be measured by various methods. ${ }^{66}$ These include quantification of TL by terminal restriction fragmentation which was one of the initial gold standard tools used for measurement and assessment of TL. Another method to assess TL is quantitative polymerase chain reaction, which provides the benefit of being able to use smaller amounts of DNA, thereby making it feasible to conduct epidemiology studies involving huge numbers of people. This method was found to have high throughput and hence is the commonly used method by most of the researchers. ${ }^{67}$ One other method uses fluorescent probes which help to quantify the average TLs as well as chromosome-specific TL. However, the disadvantage of this method is that it can only be used on mitotically active cells. Various other methods are also used to measure the TL. However, a review on measurement of TL suggested that the investigators should become familiar with the methodology, its advantages and disadvantages which will help them to select the appropriate method according to the need of the study designs. ${ }^{66}$

\section{Effect of yoga and physical activity on telomere length and telomerase activity}

Telomerase is a ribonucleic acid (RNA) containing enzyme which helps in synthesis of telomeric DNA on the ends of chromosomes. Thus, it maintains the integrity of genomes in the embryonic stem cells. Over the long term, it aids in improving the telomere maintenance. ${ }^{67}$

Various studies have evaluated the effects of physical activity and yoga on TL and telomerase activity in various conditions. ${ }^{10,59,67,68,69,70,71}$

A review on the effect of asana, pranayama and meditation on TL and telomerase activity demonstrated that asana and pranayama improve the flow of oxygen to the cells. Meditation was found to decrease the stress levels by changing the HPA axis. Hence, it can play an important role in contributing to human health and lifestyle disorders. In the review, it was found that most studies used quantitative polymerase chain reaction to quantify the TL in peripheral blood mononuclear cells (PBMCs). Following were the findings of the review. 
A pilot study was performed on patients with prostate cancer to evaluate the relation between lifestyle modifications (yoga-based stretching, breathing and meditation) and TL. The study revealed that after 5 years of lifestyle modification, there was $10 \%$ increase in TL, whereas the control group had $3 \%$ reduced TL $(p=0.03) .{ }^{72}$ There was a minor increment in the telomerase activity within 1 month followed by a decrease in the activity at 5 years. The exact treatment schedule was not available.

A prospective case control study by Krishna et al. evaluated the effect of yoga on leucocyte telomere biology and its relation with homocysteine, MDA and oxidative stress in healthy individuals. ${ }^{71}$ It was found that in individuals who practised yoga for more than 2 years, the oxidative stress was lower and TL was well preserved as compared to those who did not participate in any activity $(p<0.001)$. However, the specific type of asana or the exact yoga schedule was not known.

A study by Tolahunase ${ }^{59}$ assessed the effect of 12-week yoga and meditation-based lifestyle on cellular ageing and longevity of healthy subjects aged 30-65 years leading an unhealthy lifestyle. The intervention consisted of $90 \mathrm{~min}$ session of a set of asanas, pranayama and meditation for 5 days till 12 weeks. This prospective single-arm exploratory study showed significant improvements in 8-oxoG and reactive oxygen species (ROS) levels $(p<0.01, p<0.001)$. In addition, there was an increase in mean TL but the improvement was not significant $(p=0.069)$. Also, the telomerase activity was found to be significantly increased $(p<0.05)$ in the yoga group.

Thus, the above review indicated that yoga has a positive effect on TL. Nonetheless, there are only a few trials with very small sample sizes. Hence, there is a need for randomised trials including healthy control group and in specific diseases. ${ }^{67}$

A systematic review by Deng et al. found that the practice of physical exercise, diet, yoga and mindfulness meditation increased the telomerase activity. However, the exact mechanisms for the changes in telomerase activity have not been elucidated. Future research should include studies depicting relation between chronic psychological stress, depression, mediation and physical exercise to telomerase activation. ${ }^{73}$

\section{Physical activity and telomere length}

Various studies evaluated the effect of physical exercise on TL and DNA damage. ${ }^{68,69,70,74,75}$ Most of the studies were conducted on healthy subjects. One study that was performed on diabetic patients concluded that regular exercise (both resistance and aerobic exercise) regulates the TL and reduces the susceptibility of type $2 \mathrm{DM}$ patients to DNA damage. ${ }^{74}$ Out of all the studies, it was found that high-level physical activity resulted in longer telomere, ${ }^{68}$ whereas sedentary people with low and moderate activity showed no significant difference in TL. These effects were more in older obese people. Although the exact mechanism is not clear, it is thought that physical activity decreases chronic inflammation and oxidative stress and reduces telomeric shortening with ageing. ${ }^{75}$ The protective effects of exercise on TL are beneficial for those at risk of or living with chronic illness with huge oxidative stress and inflammation. Future studies on adults with large sample sizes are needed to be carried out.

\section{Mechanisms of action of yoga on telomere length and telomerase}

Studies have shown that yoga affects TL and telomerase activity through various mechanisms as follows:

Yogic activities that make use of diaphragmatic breathing have shown to either decrease the production of ROS or enhance the stimulation of enzymes responsible for rapid destruction of ROS or both. In one study involving yeast, it was found that elevated oxygen consumption reduced the generation and release of ROS. ${ }^{76}$ As diaphragmatic breathing improves the maximal and submaximal oxygen consumption, ${ }^{77}$ therefore pranayama can reduce the mental responses to any stimuli and hence reduce the ROS production.

Yoga is also found to increase the levels of the enzyme superoxide dismutase (SOD), which converts the superoxide anion $\mathrm{O}_{2}^{-}$(the major ROS produced in cells) into hydrogen peroxide. This, in turn, is then removed by glutathione (GSH) peroxidase or catalase, thus inhibiting the collection of ROS. This result was found in a study performed by Bhattacharya et al. on the effect of yogic breathing exercise on ROS levels. ${ }^{78}$

Another study demonstrated that yoga helps to reduce the MDAlevels (one of the primary products of lipid peroxidation by ROS) following vipassana practice, thus concluding that TL is inversely related to MDA levels in yoga group. ${ }^{79}$ Similar results were also found in another study which showed that regular practice of yoga improved the total antioxidant status (TAOS) and reduced the MDA levels, thus slowing down the shortening of leucocyte TL. ${ }^{71}$

Yogasanas also involve slow, smooth stretching of the muscles without any resistance. This sustained passive stretch improves the circulation around the tissues and organs, and thus helps in removing metabolic waste products like oxidative stress and reduces molecular damage to the cells. ${ }^{67}$

A study has also shown that yoga causes an increase in the levels of $\beta$ endorphin and sirtuin- 1 and decrease in the levels of cortisol and interleukin- 6 by producing changes in the brain through HPA axis. ${ }^{67}$ Thus, it plays an important role in reversing cellular ageing and improving cell longevity.

\section{Conclusion}

The current review demonstrates the beneficial effects of yoga and physical exercise such as aerobic and resistance 
exercise in the management of type 2 DM. Physical exercise is thought to improve muscle mass, glucose transport and insulin secretion. The beneficial effects of yoga include stress reduction, positive effects on metabolic and neuroendocrine function and inflammatory reactions. These effects are probably because of decrease in the activation of HPA axis, shift from sympathetic to parasympathetic stimulation, change in autonomic balance, mood, memory, regeneration of pancreatic cells, muscular relaxation, change in the level of hormones and neurotransmitters such as beta endorphin, serotonin, dopamine, and so on, and reduction in oxidative stress by lowering the levels of IL-6, MDA and leptin. On comparing the effects of yoga and exercise, contrasting evidences were seen. Few studies showed the superiority of yoga over physical exercise, whereas few studies demonstrated equal effectiveness of the two treatments. Yoga is found to have positive effects on TL and telomerase activity. The mechanisms include reduction in ROS, MDA and cortisol levels and increase in SOD, TAOS and $\beta$ endorphin levels in addition to improvement in blood circulation. These mechanisms, in turn, reduce the oxidative stress reversing the cellular ageing and improving cell longevity. However, most of the studies were conducted on healthy adults without control group and with small sample size. Thus, there is a need to conduct good-quality randomised controlled trials, which focus on evaluation of effects of specific standardised yoga intervention protocol on TL and telomerase activity in specific conditions like type $2 \mathrm{DM}$, with large sample size, appropriate control group and long-term follow-up. Given the increasing prevalence of type $2 \mathrm{DM}$ amongst children and adolescents, future studies also need to be conducted in different age groups (including children and adolescents). The review also suggests to conduct good-quality comparative studies to evaluate the effects of yoga over physical exercise on TL in type 2 DM in future.

\section{Acknowledgements Competing interests}

The authors have declared that no competing interests exist.

\section{Authors' contributions}

R.A.U. (Manipal Academy of Higher Education) has made substantial contribution to conception, design and acquisition of data. She has given final approval of the version to be published. R.A.U. (Saurashtra University) has been involved in conception, design, acquisition of data, drafting of the manuscript and revising it.

\section{Ethical considerations}

This article followed all ethical standards for research without direct contact with human or animal subjects.

\section{Funding}

This research received no specific grant from any funding agency in the public, commercial or not-for-profit sectors.

\section{Data availability statement}

Data sharing is not applicable to this article as no new data were created or analysed in this study.

\section{Disclaimer}

The authors admit that the article submitted is their own.

\section{References}

1. DeFronzo, Ralph A, Ferrannini $\mathrm{E}$, et al. International textbook of diabetes mellitus. 2 vol. set. 4th ed. Wiley-Blackwell; 2015, 203 p.

2. Du Diabète, FI. IDF Diabetes atlas. 8th ed. 2017, p. 16-17, International Diabetes Federation (IDF), Brussels, Belgium.

3. Fowler MJ. Microvascular and macrovascular complications of diabetes. Clin Diabetes. 2008;26:77-82. https://doi.org/10.2337/diaclin.26.2.77

4. Association AD. Standards of medical care in diabetes - 2014. Diabetes Care 2014;37(Supplement 1):S14-S80. https://doi.org/10.2337/dc14-S014

5. Innes KE, Vincent HK. The influence of yoga-based programs on risk profiles in adults with type 2 diabetes mellitus: A systematic review. Evid Based Complement Alternat Med. 2007;4(4):469-486. https://doi.org/10.1093/ecam/nel103

6. Wellen KE, Hotamisligil GS. Inflammation, stress, and diabetes. J Clin Invest. 2005;115(5):1111-1119. https://doi.org/10.1172/JCI25102

7. Rana JS, Nieuwdorp M, Jukema JW, Kastelein JJP. Cardiovascular metabolic syndrome ? an interplay of, obesity, inflammation, diabetes and coronary heart disease. Diabetes an interplay of, obesity, inflammation, diabetes and coronary heart disease. Diabetes
Obes Metab. 2007;9(3):218-232. https://doi.org/10.1111/j.1463-1326.2006.00594.x

8. Innes KE, Selfe TK. Yoga for adults with type 2 diabetes: A systematic review of controlled trials. J Diabetes Res. 2016;2016:6979370. https://doi.org/10.1155/ 2016/6979370

9. Wang J, Dong $X$, Cao L, et al. Association between telomere length and diabetes mellitus: A meta-analysis. J Int Med Res. 2016;44(6):1156-1173. https://doi. org/10.1177/0300060516667132

10. Kahl VFS, Da Silva J. Telomere length and its relation to human health In: Telomere - A complex end of a chromosome. InTech; 2016 [cited 27 Dec 2018] Available from: https://www.intechopen.com/books/telomere-a-complex-end-ofa-chromosome/telomere-length-and-its-relation-to-human-health.

11. Sampson MJ, Winterbone MS, Hughes JC, Dozio N, Hughes DA. Monocyte telomere shortening and oxidative DNA damage in type 2 diabetes. Diabetes Care. 2006;29(2):283-289. https://doi.org/10.2337/diacare.29.02.06.dc05-1715

12. Kumar V, Jagannathan A, Philip M, Thulasi A, Angadi P, Raghuram N. Role of yoga for patients with type II diabetes mellitus: A systematic review and meta-analysis. 02.001

13. Herman WH, Ye W, Griffin SJ, et al. Early detection and treatment of type 2 diabetes reduce cardiovascular morbidity and mortality: A simulation of the results of the Anglo-Danish-Dutch study of intensive treatment in people with screen-detected Anglo-Danish-Dutch study of intensive treatment in people with screen-detected
diabetes in primary care (ADDITION-Europe). Diabetes Care. 2015;38(8): diabetes in primary care (ADDITION-Europe).
1449-1455. https://doi.org/10.2337/dc14-2459

14. Li R, Zhang P, Barker LE, Chowdhury FM, Zhang X. Cost-effectiveness of interventions to prevent and control diabetes mellitus: A systematic review. Diabetes Care. 2010;33(8):1872-1894. https://doi.org/10.2337/dc10-0843

15. Chen L, Pei J-H, Kuang J, et al. Effect of lifestyle intervention in patients with type 2 diabetes: A meta-analysis. Metabolism. 2015;64(2):338-347. https://doi. org/10.1016/j.metabol.2014.10.018

16. Micozzi MS, Koop CE, Charles E, Lundberg GD. Fundamentals of complementary and alternative medicine. St. Louis, MO: Saunders/Elsevier; 2011, 717 p.

17. Pandey A, Tripathi P, Pandey R, Srivatava R, Goswami S. Alternative therapies usefu in the management of diabetes: A systematic review. J Pharm Bioallied Sci. 2011;3(4):504-512. https://doi.org/10.4103/0975-7406.90103

18. Cui J, Yan J-H, Yan L-M, Pan L, Le J-J, Guo Y-Z. Effects of yoga in adults with type 2 diabetes mellitus: A meta-analysis. J Diabetes Investig. 2017;8(2):201-209. https://doi.org/10.1111/jdi.12548

19. Raveendran AV, Deshpandae A, Joshi SR. Therapeutic role of yoga in type 2 diabetes. Endocrinol Metab. 2018:33:307-317. https://doi.org/10.3803/EnM. 2018.33.3.307

20. Dash S, Thakur AK. Effect of yoga in patient's with Type-II diabetes mellitus. J Evol Med Dent Sci. 2014;3(7):1642-1655. https://doi.org/10.14260/jemds/2014/2038

21. Singh V, Sherpa N, Khandelwal B. Psycho-neuro-endocrine-immune mechanisms of action of yoga in type II diabetes. Anc Sci Life. 2015;35(1):12. https://doi. org/10.4103/0257-7941.165623

22. Thind $H$, Lantini $R$, Balletto $B L$, et al. The effects of yoga among adults with type 2 diabetes: A systematic review and meta-analysis. Prev Med (Baltim). 2017;105(April):116-126. https://doi.org/10.1016/j.ypmed.2017.08.017

23. Sharma D, Sharma D, Sharma D, Gupta M, Meena M. Effect of yoga on blood glucose and glycosylated haemoglobin level in diabetes mellitus type-2 patients. Int J Med Sci. 2015;2(1)

24. Jyotsna V. Prediabetes and type 2 diabetes mellitus: Evidence for effect of yoga. Indian J Endocrinol Metab. 2014;18(6):745. https://doi.org/10.4103/2230-8210. 141318 
25. Satish L, Lakshmi Vs. Impact of individualized yoga therapy on perceived quality of life performance on cognitive tasks and depression among Type II diabetic patients. Int J Yoga. 2016;9(2):130. https://doi.org/10.4103/0973-6131.183707

26. Edwards J, Hosseinzadeh $\mathrm{H}$. The impact of structured physical activity on glycaemic control in diabetes prevention programmes: A systematic review. Proc Singapore Healthc. 2018;27(3):193-204. https://doi.org/10.1177/2010105817739924

27. Hemmingsen B, Gimenez-Perez G, Mauricio D, Roqué i Figuls M, Metzendorf M-I, Richter B. Diet, physical activity or both for prevention or delay of type 2 diabetes mellitus and its associated complications in people at increased risk of developin type 2 diabetes mellitus. Cochrane Database Syst Rev. 2017/12(12):CD003054. https://doi.org/10.1002/14651858.CD003054.pub4

28. Colberg SR. Key Points from the updated guidelines on exercise and diabetes. Front Endocrinol (Lausanne). 2017;8:33. https://doi.org/10.3389/fendo.2017.00033

29. Hamasaki H. Daily physical activity and type 2 diabetes: A review. World J Diabetes. 2016;7(12):243-251. https://doi.org/10.4239/wjd.v7.i12.243

30. Pai L-W, Li T-C, Hwu Y-J, Chang S-C, Chen L-L, Chang P-Y. The effectiveness of regular leisure-time physical activities on long-term glycemic control in people with type 2 diabetes: A systematic review and meta-analysis. Diabetes Res Clin Pract. 2016;113:77-85. https://doi.org/10.1016/j.diabres.2016.01.011

31. Williams JE, Helsel B, Nelson B, Eke R. Exercise considerations for type 1 and type 2 diabetes. ACSM's Heal Fit J. 2018;22(1):10-16. https://doi.org/10.1249/FIT 0000000000000359

32. Thomas D, Elliott EJ, Naughton GA. Exercise for type 2 diabetes mellitus. Cochrane Database Syst Rev. 2006;3:CD002968. https://doi.org/10.1002/14651858.CD00 2968.pub2

33. Malkawi AM. The effectiveness of physical activity in preventing type 2 diabetes in high risk individuals using well-structured interventions: A systematic review. J Diabetol. 2012;3(2):5.

34. Hemmingsen B, Gimenez-Perez G, Mauricio D, Roqué I, Figuls M, Metzendorf M-I, Richter B. Diet, physical activity or both for prevention or delay of type 2 diabetes mellitus and its associated complications in people at increased risk of developing type 2 diabetes mellitus. Cochrane Database Syst Rev. 2017;12(12):CD003054. https://doi.org/10.1002/14651858.CD003054.pub4

35. Sigal RJ, Kenny GP, Wasserman DH, Castaneda-Sceppa C, White RD. Physical activity/exercise and type 2 diabetes. Diabetes Care. 2006;29(6):1433-1438. https://doi.org/10.2337/dc06-9910

36. Chen S-C, Ueng K-C, Lee S-H, Sun K-T, Lee M-C. Effect of T'ai Chi exercise on biochemical profiles and oxidative stress indicators in obese patients with type 2 diabetes. J Altern Complement Med. 2010;16(11):1153-1159. https://doi.org/ 10.1089/acm.2009.0560

37. Sato $Y$, Nagasaki M, Nakai N, Fushimi T. Physical exercise improves glucose metabolism in lifestyle-related diseases. Exp Biol Med (Maywood). 2003; 228(10):1208-1212. https://doi.org/10.1177/153537020322801017

38. Qiu S, Cai X, Schumann U, Velders M, Sun Z, Steinacker JM. Impact of walking on glycemic control and other cardiovascular risk factors in type 2 diabetes: A metaanalysis. Manzoli L, editor. PLoS One. 2014;9(10):e109767. https://doi.org/10. 1371/journal.pone.0109767

39. Nieuwoudt S, Fealy CE, Foucher JA, et al. Functional high-intensity training improves pancreatic $\beta$-cell function in adults with type 2 diabetes. Am J Physio Metab. 2017;313(3):E314-E320. https://doi.org/10.1152/ajpendo.00407.2016

40. Advika TS, Idiculla J, Kumari SJ. Exercise in patients with Type 2 diabetes: Facilitators and barriers - A qualitative study. J Fam Med Prim Care. 2017;6(2): 288-292. https://doi.org/10.4103/2249-4863.219998

41. Adeniyi AF, Anjana RM, Weber MB. Global account of barriers and facilitators of physical activity among patients with diabetes mellitus: A narrative review of the literature. Curr Diabetes Rev. 2016;12(4):440-448. https://doi.org/10.2174/1573 399812666160609102956

42. Miyauchi M, Toyoda M, Kaneyama N, et al. Exercise therapy for management of type 2 diabetes mellitus: Superior efficacy of activity monitors over pedometers. Diabetes Res. 2016;2016:1-7. https://doi.org/10.1155/2016/5043964

43. Sigal RJ, Kenny GP, Wasserman DH, Castaneda-Sceppa C. Physical activity/exercise and type 2 diabetes. Diabetes Care. 2004;27(10):2518-2539. https://doi.org/ 10.2337/diacare.27.10.2518

44. Dela F, Ploug T, Handberg A, et al. Physical training increases muscle GLUT4 protein and mRNA in patients with NIDDM. Diabetes. 1994;43(7):862-865. https://doi.org/10.2337/diab.43.7.862

45. Krotkiewski M, Lönnroth $P$, Mandroukas K, et al. The effects of physical training on insulin secretion and effectiveness and on glucose metabolism in obesity and type 2 (non-insulin-dependent) diabetes mellitus. Diabetologia. 1985;28(12):881-890. 2 (non-insulin-dependent) diabetes mell
https://doi.org/10.1007/BF00703130

46. Fishman L, Saltonstall E. Yoga in pain management. In: Integrative pain medicine. Totowa, NJ: Humana Press; 2008, p. 259-284. https://doi.org/10.1007/978-159745-344-8_13

47. Govindaraj R, Karmani S, Varambally S, Gangadhar BN. Yoga and physical exercise - A review and comparison. Int Rev Psychiatry. 2016;28(3):242-253. https://doi.org/10.3109/09540261.2016.1160878

48. Aljasir B, Bryson M, Al-Shehri B. Yoga practice for the management of type I diabetes mellitus in adults: A systematic review. Evid Based Complement Alternat Med. 2010;7(4):399-408. https://doi.org/10.1093/ecam/nen027

49. Jayawardena R, Ranasinghe P, Chathuranga T, Atapattu PM, Misra A. The benefits of yoga practice compared to physical exercise in the management of type 2 Diabetes Mellitus: A systematic review and meta-analysis. Diabetes Metab Synd Clin Res Rev. 2018;12(5):795-805. https://doi.org/10.1016/j.dsx.2018.04.008

50. Malhotra $V$, Singh $S$, Singh $P$, et al. Effects of yoga asanas and pranayama in noninsulin dependent diabetes mellitus. Indian J Tradit Know. 2004;3:162-167.
51. Singh S, Kyizom T, Singh KP, Tandon OP, Madhu S V. Influence of pranayamas and yoga-asanas on serum insulin, blood glucose and lipid profile in type 2 diabetes. Indian J Clin Biochem. 2008;23(4):365-368. https://doi.org/10.1007/s12291008-0080-9

52. Deshmukh SV, Bedekar N. Yoga therapy as potential treatment for type II diabetic patients. Deshmukh, Supriya Vinay Bedekar, Nilima. 2015;2(1):1-7.

53. Chimkode SM. Effect of yoga on blood glucose levels in patients with type 2 diabetes mellitus. J Clin Diagnostic Res. 2015;34(10):2208-2210. https://doi. org/10.7860/JCDR/2015/12666.5744

54. Jyotsna V, Ambekar S, Dhawan A, Joshi A, Kumar N, Sreenivas V. Comprehensive yogic breathing program improves quality of life in patients with diabetes. Indian $J$ Endocrinol Metab. 2012;16(3):423. https://doi.org/10.4103/2230-8210.95692

55. Kiecolt-Glaser JK, Christian LM, Andridge R, et al. Adiponectin, leptin, and yoga practice. Physiol Behav. 2012;107(5):809-813. https://doi.org/10.1016/j.physbeh. 2012.01.016

56. Newberg $A B$, Iversen J. The neural basis of the complex mental task of meditation: Neurotransmitter and neurochemical considerations. Med Hypotheses. 2003;61(2):282-291. https://doi.org/10.1016/S0306-9877(03)00175-0

57. Shree N, Bhonde RR. Can yoga therapy stimulate stem cell trafficking from bone marrow? J Ayurveda Integr Med. 2016;7(3):181-184. https://doi.org/10.1016/j jaim.2016.07.003

58. Hedge SV, Pinto VJ, Adhikari P, D'Souza S, Kotlan S, D'Souza V. Effect of 3-month yoga on oxidative stress in type 2 diabetes with or without complications a controlled clinical trial. Diabetes Care. 2011;34:2208-2210. https://doi.org/10.2337/dc10-2430

59. Tolahunase M, Sagar R, Dada R. Impact of yoga and meditation on cellular aging in apparently healthy individuals: A prospective, open-label single-arm exploratory study. 2017;2017(4):1-9. https://doi.org/10.1155/2017/2784153

60. Pace TWW, Negi LT, Adame DD, et al. Effect of compassion meditation on neuroendocrine, innate immune and behavioral responses to psychosocial stress. Psychoneuroendocrinology. 2009;34(1):87-98. https://doi.org/10.1016/j. psyneuen.2008.08.011

61. Harte JL, Eifert GH, Smith R. The effects of running and meditation on betaendorphin, corticotropin-releasing hormone and cortisol in plasma, and on mood.
Biol Psychol. 1995:40(3):251-265. https://doi.org/10.1016/0301-0511(95)05118-T Biol Psychol. 19. 62. McClintock B. The sta
1941;26(2):234-282.

63. Muller HJ. The remaking of chromosomes. Collecting Net. 1938;8:182-195.

64. Blackburn EH. Structure and function of telomeres. Nature. 1991;350(6319): 569-573. https://doi.org/10.1038/350569a0

65. Zakian VA. Structure, function, and replication of Saccharomyces cerevisiae telomeres. Annu Rev Genet. 1996;30(1):141-172. https://doi.org/10.1146/ annurev.genet.30.1.141

66. Montpetit AJ, Alhareeri AA, Montpetit M, et al. Telomere length. Nurs Res. 2014;63(4):289-299. https://doi.org/10.1097/NNR.0000000000000037

67. Rathore $\mathrm{M}$, Abraham J. Implication of asana, pranayama and meditation on telomere stability. Int J Yoga. 2018;11(3):186-193. https://doi.org/10.4103/ijoy. IJOY_51_17

68. Tucker LA. Physical activity and telomere length in U.S. men and women: An NHANES investigation. Prev Med (Baltim). 2017;100:145-151. https://doi. org/10.1016/j.ypmed.2017.04.027

69. Shadyab AH, LaMonte MJ, Kooperberg C, et al. Association of accelerometermeasured physical activity with leukocyte telomere length among older women. Gerontol Ser A. 2017;72(11):1532-1537. https://doi.org/10.1093/gerona/glx037

70. Shadyab AH, LaMonte MJ, Kooperberg C, et al. Leisure-time physical activity and leukocyte telomere length among older women. Exp Gerontol. 2017;95:141-147. https://doi.org/10.1016/j.exger.2017.05.019

71. Krishna BH. Association of leukocyte telomere length with oxidative stress in yoga practitioners. J Clin Diagnostic Res. 2015;16(7):457-464. https://doi.org/10.7860/ JCDR/2015/13076.5729

72. Ornish D, Lin J, Chan JM, et al. Effect of comprehensive lifestyle changes on telomerase activity and telomere length in men with biopsy-proven low-risk prostate cancer: 5-year follow-up of a descriptive pilot study. Lancet Oncol. 2013;14(11):1112-1120. https://doi.org/10.1016/S1470-2045(13)70366-8

73. Deng W, Cheung ST, Tsao SW, Wang XM, Tiwari AFY. Telomerase activity and its association with psychological stress, mental disorders, lifestyle factors and
interventions: A systematic review. Psychoneuroendocrinology. 2016;64:150-163. https://doi.org/10.1016/j.psyneuen.2015.11.017

74. Dimauro I, Sgura A, Pittaluga M, et al. Regular exercise participation improves genomic stability in diabetic patients: An exploratory study to analyse telomere length and DNA damage. Sci Rep. 2017;7(1):4137. https://doi.org/10.1038/ s41598-017-04448-4

75. Arsenis NC, You T, Ogawa EF, Tinsley GM, Zuo L. Physical activity and telomere length: Impact of aging and potential mechanisms of action. Oncotarget. 2015;8(27):45008-45019. https://doi.org/10.18632/oncotarget.16726

76. Barros $\mathrm{MH}$, Bandy B, Tahara EB, Kowaltowski AJ. Higher respiratory activity decreases mitochondrial reactive oxygen release and increases life span in
Saccharomyces cerevisiae. J Biol Chem. 2004;279(48):49883-49888. https://doi. Saccharomyces cerevisiae. J Biol
org/10.1074/jbc.M408918200

77. Shaw I, Shaw BS, Brown GA. Role of diaphragmatic breathing and aerobic exercise in improving pulmonary function and maximal oxygen consumption in asthmatics. Sci Sports. 2010;25(3):139-145. https://doi.org/10.1016/j.scispo.2009.10.003

78. Bhattacharya S, Pandey US, Verma NS. Improvement in oxidative status with yogic breathing in young healthy males. Indian J Physiol Pharmacol. 2002;46(3):349-354

79. Ingole A, Bahattare V, Chaware S. Reducing ucing oxidative stress through Vipassana meditation. Int Med J. 2015;2(April):189-191. 